\title{
Livšic's theorem for semisimple Lie groups
}

\author{
MATTHEW NICOL $\dagger$ and MARK POLLICOTT $\dagger$ \\ $\dagger$ Department of Mathematics and Statistics, University of Surrey, \\ Guildford GU2 7XH, UK \\ $\ddagger$ Mathematics Department, University of Manchester, Oxford Rd, \\ Manchester M13 9PL, UK \\ (e-mail:mp@ma.man.ac.uk)
}

(Received 20 November 1998 and accepted in revised form 8 August 2000)

\begin{abstract}
In this paper we show that strong generalizations of the measurable Livšic theorem for cocycles taking values in connected non-compact linear semisimple Lie groups, a canonical example being $S L(2, \mathbb{R})$, can be deduced from an elegant approach of Brin and Pesin to the dynamics of partially hyperbolic systems.
\end{abstract}

\section{Introduction}

Let $T: M \rightarrow M$ be a transitive Anosov diffeomorphism of a compact Riemannian manifold and let $\mu$ be a $T$-invariant probability measure which is equivalent to Lebesgue measure. Let $g: M \rightarrow G$ be a $C^{1}$ function taking values in a finite-dimensional connected matrix Lie group $G$ with norm $\|\cdot\|$ and metric $d$. If $G$ is a matrix group then we define the norm of $A \in G$ by $\|A\|^{2}=\operatorname{Trace}\left(A \bar{A}^{\mathrm{t}}\right)$ for $A \in G$.

In this paper we are interested in measurable solutions $h: M \rightarrow G$ to the equation

$$
h(T x)=g(x) h(x), \quad \mu \text { a.e. }
$$

In this context we say that $h$ is a coboundary for $g$. In particular, we are interested in conditions under which a measurable coboundary $h$ must have a Hölder continuous version $h^{\prime}$ such that $h=h^{\prime} \mu$ a.e.

Livšic [9] (see also $[\mathbf{6}, \mathbf{8}, \mathbf{1 2}, \mathbf{1 6}]$ ) studied the corresponding equation for a continuous real-valued cocycle satisfying a Dini property over a transitive Anosov diffeomorphism preserving a smooth measure. He showed that a measurable solution $h$ to equation (1.1) has a continuous version.

Quas [17] proved analogous results for essentially bounded, real-valued coboundaries of continuous cocycles $g$ where the base dynamics was a locally eventually onto continuous map or a minimal system, such as an irrational rotation of $S^{1}$.

Nicol and Pollicott [10] extended this result to continuous cocycles taking values in Lie groups with non-positive sectional curvature (and the same assumptions on the base 
dynamics). They also showed that essentially bounded measurable cocycles taking values in subgroups of $G L\left(n, \mathbb{R}\right.$ ) (with base dynamics a transitive $C^{\infty}$ Anosov diffeomorphism equipped with a measure equivalent to Lebesgue) are continuous. This paper (using a very different technique) removes the essentially bounded assumption-if a pinching condition on $g$ and a smooth measure in the base are assumed.

Hypothesis 1. Assume that $G$ satisfies the condition that there exist two cocompact lattices $\Gamma, \Gamma^{\prime}$ (i.e. $G / \Gamma$ and $G / \Gamma^{\prime}$ are compact homogeneous spaces) such that $\Gamma \cap \Gamma^{\prime}=\{e\}$.

This property holds for $G=\mathbb{R}^{n}, S L(2, \mathbb{R})$, and, more generally, connected noncompact linear semisimple Lie groups. The proof of this fact, suggested to us by S. Mozes and S. G. Dani, is given in Appendix A. It is false, however, in the case of the Heisenberg group.

We recall that a diffeomorphism $f$ of a compact Riemannian manifold $M$ is Anosov if there exists a $D f$-invariant splitting $T M=E^{u} \oplus E^{s}, C>0$ and $0<\lambda<1$ such that

$$
\left\|D f^{n}(v)\right\| \leq C \lambda^{n}\|v\|, \quad \text { for } v \in E^{s}
$$

and

$$
\left\|D f^{-n}(v)\right\| \leq C \lambda^{n}\|v\|, \quad \text { for } v \in E^{u},
$$

where $n \geq 0$. We assume that $T$ is Anosov, transitive and has an invariant measure $\mu$ which is equivalent to Lebesgue.

Recall that the adjoint map Ad : $G \rightarrow \operatorname{Aut}(L G)$ is the automorphism of the Lie algebra $L G$ defined so that $\operatorname{Ad}(g)$ is the derivative of conjugation by $g \in G$.

Hypothesis 2. We assume that $g: M \rightarrow G$ satisfies the pinching condition: $\lambda<$ $\|\operatorname{Ad}(g(x))\|<1 / \lambda$ for all $x \in M$.

This property ensures that the group extension is a partially hyperbolic dynamical system (see Definition 2.1).

Our main result is the following.

THEOREM 1.1. If $G$ is a connected non-compact semisimple Lie group and for all $x \in M$

$$
\lambda<\|\operatorname{Ad}(g(x))\|<\frac{1}{\lambda}
$$

then any measurable solution $h: M \rightarrow G$ to equation (1.1) must be equal to a Hölder continuous function a.e.

COROLlaRY 1.2. If $g: M \rightarrow S L(2, \mathbb{R})$ and for all $x \in M$

$$
\lambda<\|\operatorname{Ad}(g(x))\|<\frac{1}{\lambda}
$$

then any measurable solution $h: M \rightarrow S L(2, \mathbb{R})$ to the equation $(1.1)$ must be equal to a Hölder continuous function $\mu$ a.e.

The following result of Niţică and Török [11, Theorem 4, p. 9] strengthens the regularity of $h(x)$ in equation (1.1). 
Proposition 1.3. If $g: M \rightarrow G$ is $C^{k}$ and $h$ is a continuous solution to equation 1.1 then $h$ is $C^{k-1}$.

Combined with Theorem 1.1 this has the following corollary.

COROLlary 1.4. If $G$ satisfies Hypothesis 1 and $g$ is $C^{k}$ and satisfies Hypothesis 2 then any measurable solution $h: M \rightarrow G$ to equation (1.1) must be equal to a $C^{k-1}$ function $\mu$ a.e. In particular, if $g$ is $C^{\infty}$, then $h$ has a $C^{\infty}$ version $\mu$ a.e.

We shall base our proof of the theorem on Brin and Pesin's analysis of partially hyperbolic diffeomorphisms on compact manifolds [2] and Brin's work [1] on frame flows.

In $\$ 2$ we review these results on partially hyperbolic systems, which are a generalization of the classical theory of Anosov systems. In $\S 3$ we prove our main result.

\section{Partially hyperbolic systems}

Suppose that $T: M \rightarrow M$ is a transitive Anosov diffeomorphism of a compact Riemannian manifold preserving a smooth invariant measure $\mu$.

There is a well known theory concerning the properties of stable and unstable manifolds defined by

$$
\begin{gathered}
W^{s}(x):=\left\{y: d\left(T^{n}(x), T^{n}(y)\right) \rightarrow 0 \text { as } n \rightarrow \infty\right\} \\
W^{u}(x):=\left\{y: d\left(T^{n}(x), T^{n}(y)\right) \rightarrow 0 \text { as } n \rightarrow-\infty\right\} .
\end{gathered}
$$

In particular, for each $x \in M$, there are $C^{1}$ immersed submanifolds $W^{u}(x), W^{s}(x) \subset M$ with $T_{x} W^{s}(x)=E_{x}^{s}$ and $T_{x} W^{u}(x)=E_{x}^{u}$.

Definition 2.1. We say $\hat{T}: N \rightarrow N$ is a partially hyperbolic diffeomorphism of a compact Riemannian manifold $N$ if there is a splitting $T N=E^{u} \oplus E^{s} \oplus E^{0}$ and constants $C>0$ and $\lambda, \rho_{1}, \rho_{2}$ satisfying

$$
0<\lambda<\rho_{1}<1<\rho_{2}<\frac{1}{\lambda}
$$

and such that $\left\|\left.D \hat{T}^{n}\right|_{E^{s}}\right\| \leq C \lambda^{n},\left\|\left.D \hat{T}^{-n}\right|_{E^{u}}\right\| \leq C \lambda^{n}$ and $C \rho_{1}^{n} \leq\left\|\left.D \hat{T}^{n}\right|_{E^{0}}\right\| \leq C \rho_{2}^{n}$ for $n \geq 0$ (where $\|\cdot\|$ is the norm on $T N$ ).

We shall be interested in partially hyperbolic diffeomorphisms which arise as homogeneous extensions of Anosov diffeomorphisms of a compact Riemannian manifold $M$ satisfying $\left\|\left.D \hat{T}^{n}\right|_{E^{s}}\right\| \leq C \lambda^{n},\left\|\left.D \hat{T}^{-n}\right|_{E^{u}}\right\| \leq C \lambda^{n}$ for all $n \geq 0$. Suppose that $\Gamma$ is a cocompact lattice of $G$. Then $H:=G / \Gamma$ is a compact homogeneous space with a left invariant Haar measure $v$.

We consider the compact manifold $N=M \times H$ and the diffeomorphism $\hat{T}: N \rightarrow N$ given by $\hat{T}(x, h \Gamma)=(T x, g(x) h \Gamma)$, which preserves the measure $\hat{\mu}=\mu \times \nu$.

We assume that the cocycle $g$ satisfies

$$
\rho_{1} \leq\|\operatorname{Ad}(g(x))\| \leq \rho_{2}
$$

for all $x \in M$ where $\lambda<\rho_{1}<1<\rho_{2}<1 / \lambda$ (where $\|\cdot\|$ is, with abuse of notation, the operator norm of $\operatorname{Ad}(g): L G \rightarrow L G)$. Under these assumptions $\hat{T}: N \rightarrow N$ is a $C^{1}$ partially hyperbolic diffeomorphism. 
The following result extends the familiar results on stable and unstable manifolds for Anosov diffeomorphisms to the context of partially hyperbolic diffeomorphisms.

Proposition 2.2. (Brin and Pesin [2], Hirsch et al. [4]) (i) Let $\hat{T}: N \rightarrow N$ be a $C^{1}$ partially hyperbolic diffeomorphism. For each $z \in N$ there are $C^{1}$ immersed stable and unstable submanifolds $W^{s}(z), W^{u}(z)$, and a center submanifold $W^{0}(z) \subset M$ with $T_{z} W^{s}(z)=E_{z}^{s}, T_{z} W^{u}(z)=E_{z}^{u}$ and $T_{z} W^{0}(x)=E_{z}^{0}$.

(ii) The foliations $\left\{W^{s}(z)\right\},\left\{W^{u}(z)\right\}$ and $\left\{W^{0}(z)\right\}$ are each Hölder continuous.

Remark 2.3. In general the foliations may have only Hölder regularity, even though $\hat{T}$ is $C^{\infty}[7]$.

Proposition 2.4. (Brin [1, p. 170]) Let $\hat{T}: N \rightarrow N$ be a $C^{1}$ partially hyperbolic diffeomorphism preserving a measure $\hat{\mu}$ equivalent to Lebesgue and suppose that $A \subset N$ is $\hat{T}$ invariant. Then A consists up to a set of $\hat{\mu}$ measure zero $(\bmod 0)$, of the union of stable and unstable manifolds for $\hat{T}$.

\section{Proof of Theorem 1.1}

Since $G$ satisfies Hypothesis 1 we may choose discrete subgroups $\Gamma, \Gamma^{\prime}<G$ satisfying the conditions of Hypothesis 1 . Note that by assumption $G / \Gamma, G / \Gamma^{\prime}$ are compact homogeneous spaces.

Define $H:=G / \Gamma$. We now consider the compact manifold $N=M \times H$ and the diffeomorphism $\hat{T}: N \rightarrow N$ given by $\hat{T}(x, g \Gamma)=(T x, g(x) g \Gamma)$. For ease of notation we will write $(x, g \Gamma)$ as $(x, g)$. Note that the Haar measure on $G$ (see [3]) induces a measure $v_{\Gamma}$ on $G / \Gamma$, an induced metric which we will write as $d_{H}$ and that $\hat{T}(x, g)=(T x, g(x) g)$ preserves $\mu \times v_{\Gamma}$. We will use the notation $d_{M}$ to refer to the metric in the base $M$. The measurable map $h: M \rightarrow G$ induces a measurable map $h: M \rightarrow H$ (we will use the same notation for both maps when it is clear which is meant).

Given $g \in G$, define the measurable sets

$$
\Lambda_{g}=\{(x, h(x) g): x \in M\} \subset M \times H .
$$

Each set $\Lambda_{g}$ is invariant under the skew product

$$
\begin{aligned}
& \hat{T}: M \times H \rightarrow M \times H \\
& \hat{T}(x, g)=(T x, g(x) g) .
\end{aligned}
$$

This is clear since

$$
\hat{T}(x, h(x) g)=(T x, g(x) h(x) g)=(T x, h(T x) g),
$$

as $h(T x)=g(x) h(x) \mu$ a.e.

Given small $\delta>0$, let $U(\delta)$ be a neighborhood of the coset of the identity element in $H$ of diameter equal to $\delta$ in the induced metric.

Consider the set $\Lambda_{U(\delta)}=\left\{\Lambda_{g}: g \in U(\delta)\right\}$. Note that $\left(\mu \times \nu_{\Gamma}\right)\left(\Lambda_{U(\delta)}\right)>0$ and $\Lambda_{U(\delta)}$ is measurable with respect to the ergodic decomposition of $\hat{T}$. Thus by [1, p. 170] the set $\Lambda_{U(\delta)}$ consists up to a set of measure zero of entire stable and unstable manifolds for $\hat{T}$. 
Without loss of generality, by adding or removing a set of zero measure, we can assume that $\Lambda_{U(\delta)}$ is simply a union of stable and unstable manifolds.

By Proposition 2.2(ii) the foliations of $M \times H$ into stable $\left\{W^{s}(x, \gamma)\right\}$ and unstable $\left\{W^{u}(x, \gamma)\right\}$ manifolds are Hölder continuous for some Hölder exponent ( $\alpha$ say) and Hölder constant (C say). This means that for any $(x, g) \in M \times H$ there is a Hölder continuous map $\Pi_{x}^{s}: W^{s}(x) \rightarrow W^{s}(x, g)$, and similarly there is a Hölder continuous map $\Pi_{x}^{u}: W^{u}(x) \rightarrow W^{u}(x, g)$.

It is a standard result [5] that there exists $\delta^{\prime}>0$ such that if $d_{M}(x, y)<\delta^{\prime}$ then there exists a unique point $x^{\prime} \in M$ with $W^{s}(x) \cap W^{u}(y)=\left\{x^{\prime}\right\}$, i.e. there is a 'local product structure'.

Consider those $x, y \in M$ for which $h(x)$ and $h(y)$ are well defined and suppose that $d_{M}(x, y)=\delta<\delta^{\prime}$. Since we know that $W^{s}(x, h(x) \gamma) \subset \Lambda_{U(\delta)}$, the above result on the Hölder nature of the foliations shows that there exists $\gamma^{\prime}=\gamma^{\prime}\left(x^{\prime}\right) \in H$ such that $\left(x^{\prime}, h\left(x^{\prime}\right) \gamma^{\prime}\right) \in W^{s}(x, h(x) \gamma)$. Similarly, there exists $\gamma^{\prime \prime}=\gamma^{\prime \prime}(y) \in H$ such that $\left(y, h(y) \gamma^{\prime \prime}\right) \in W^{u}\left(x^{\prime}, h\left(x^{\prime}\right) \gamma^{\prime}\right)$.

Since the foliations $W^{s}(x, \gamma)$ and $W^{u}(x, \gamma)$ are $C^{1}$ immersed submanifolds which are Hölder continuous in $(x, \gamma)$, we can use the triangle inequality to estimate

$$
d\left((x, h(x) \gamma),\left(y, h(y) \gamma^{\prime \prime}\right)\right)<C d_{M}(x, y)^{\alpha}<C \delta^{\alpha} .
$$

By the triangle inequality

$$
d_{H}(h(x), h(y)) \leq d_{H}(h(x), h(x) \gamma)+d_{H}\left(h(x) \gamma, h(y) \gamma^{\prime \prime}\right)+d_{H}\left(h(y) \gamma^{\prime \prime}, h(y)\right) .
$$

Moreover, since the map $\gamma \mapsto f(\gamma):=\sup _{g \in H} d_{H}(g, g \gamma)$ is continuous, for any $\epsilon>0$ we can assume $\delta$ was chosen sufficiently small that $f(\gamma)<\epsilon$ if $\gamma \in U(\delta)$. In particular, we conclude that $d_{H}(h(x), h(y)) \leq 2 \epsilon+C \delta^{\alpha}$. Since $\epsilon$ is arbitrary, we see that $x \mapsto h(x)$ is continuous (in fact Hölder continuous).

We wish to conclude the continuity of $h: M \rightarrow G$, rather than $h: M \rightarrow H$. We will denote by $\bar{h}(x)$ the value of $h: M \rightarrow G$ and $h_{H}(x)$ the possible values (in $G$ ) of $h: M \rightarrow H$, i.e. $h_{H}(x)=\{\bar{h}(x) \gamma: \gamma \in \Gamma\}$ is a multivalued function with continuous branches and $h(x) \in h_{H}(x)$. It remains to show that $h(x)$ which, a priori, could change branches in a measurable way does, in fact, involve a single constant choice.

Now we can use Hypothesis 2 and the second cocompact lattice $\Gamma^{\prime}$. Define $H^{\prime}:=G / \Gamma^{\prime}$ and repeat the construction given above.

Note that by Hypothesis 1 the sets $h_{H}(x)=\{\bar{h}(x) \gamma: \gamma \in \Gamma\}$ and $h_{H^{\prime}}(x)=\left\{\bar{h}(x) \gamma^{\prime}\right.$ : $\left.\gamma^{\prime} \in \Gamma^{\prime}\right\}$ have intersection only when $\gamma=\gamma^{\prime}=e$. Thus there is a well-defined solution $h(x)$ which, by local considerations, is Hölder continuous.

\section{Related results}

It is interesting to ask whether the generalization of this result holds for the identity

$$
h(T x) f(x)=h(x) g(x), \quad \mu \text { a.e. }
$$

In this case we say that $f$ and $g$ are cohomologous via $h$. In fact $h$ induces a conjugacy between extensions with cocycles $f$ and $g$, respectively, which preserves the fibre $G$. 
A positive answer to this question would imply that a measurable conjugacy (which preserves the fibre) between extensions with cocycles $f$ and $g$, respectively, is necessarily continuous.

However, Walkden [20] has shown that if $G$ is non-connected and non-compact then there exist smooth cocycles $f, g$ which are cohomologous via a measurable cobounding function $h$ which has no continuous version.

In the context of cohomologous cocycles $f, g$ we may arrange that $h$ is $C^{0}$ but not $C^{\alpha}$. However, in the case of a coboundary if $g(x)=h(T x) h(x)^{-1}$ then $h$ has a $C^{k-1}$ version if $g$ is $C^{k}$.

Consider a linear hyperbolic toral automorphism $T: \mathbb{R}^{4} / \mathbb{Z}^{4} \rightarrow \mathbb{R}^{4} / \mathbb{Z}^{4}$ defined by a matrix of the form

$$
\left(\begin{array}{ll}
A & 0 \\
0 & B
\end{array}\right) \in S L(4, \mathbb{Z})
$$

where $A, B \in S L(2, \mathbb{Z})$.

By structural stability, we know that for any nearby diffeomorphism $S: \mathbb{R}^{4} / \mathbb{Z}^{4} \rightarrow$ $\mathbb{R}^{4} / \mathbb{Z}^{4}$ there exists a unique conjugating homeomorphism $\pi: \mathbb{R}^{4} / \mathbb{Z}^{4} \rightarrow \mathbb{R}^{4} / \mathbb{Z}^{4}$ (i.e. $T \circ \pi=\pi \circ S$ ).

We can assume that $S$ is of the form $S(x, y)=(A x, B y+v \phi(x))$, where $B v=\lambda v$, $v$ is the maximal eigenvector and $\phi: \mathbb{R}^{4} / \mathbb{Z}^{4} \rightarrow \mathbb{R}$ is some $C^{\infty}$ function, sufficiently small in the $C^{0}$ topology to ensure the existence of a unique conjugating homeomorphism. The conjugacy takes the form $\pi(x, y)=(x, y+\psi(x) v)$. In particular, $\psi(x)$ satisfies

$$
\lambda \psi(x)-\psi(A x)=\phi(x) .
$$

An argument based on Fourier series similar to that given by de la Llave [7, Theorem 6.3] shows that given $\alpha$ we may choose $A$ and $B$ so that $\psi$ is $C^{1}$ but not $C^{1+\alpha}$.

Thus the conjugating homeomorphism is differentiable (with derivative $D \pi$ ) and we can clearly differentiate the conjugating identity to write

$$
(D T) \circ \pi \cdot(D \pi)=(D \pi) \circ S \cdot(D S) .
$$

Since $T$ is linear, we have that $D T$ is constant on $\mathbb{R}^{4} / \mathbb{Z}^{4}$ and thus $(D T) \circ \pi=D T$, and so if we define

$$
\begin{aligned}
& g=D T: \mathbb{R}^{4} / \mathbb{Z}^{4} \rightarrow S L(4, \mathbb{R}) \\
& h=D \pi: \mathbb{R}^{4} / \mathbb{Z}^{4} \rightarrow S L(4, \mathbb{R})
\end{aligned}
$$

and

$$
f=D T: \mathbb{R}^{4} / \mathbb{Z}^{4} \rightarrow G L(4, \mathbb{R})
$$

then

$$
g(x) h(x)=h(S x) f(x)
$$

where $h(x)$ is continuous but not $C^{\alpha}$.

We can hope to replace the hypothesis that there exists a measurable solution $h(x)$ by an intrinsic property of $f$. Let $G$ have metric $d$. Let $(T, X, \mu)$ be a mixing subshift of 
finite type. We call $d$ a Lipschitz metric if $G$ is complete with respect to $d$ and if there exists for every $g \in G$ a constant $\alpha_{g}$ such that the following condition holds:

$$
\begin{aligned}
\frac{1}{\alpha_{g}} & \leq \min \left\{d\left(g h, g h^{\prime}\right), d\left(h g, h^{\prime} g\right)\right\} \\
& \leq \max \left\{d\left(g h, g h^{\prime}\right), d\left(h g, h^{\prime} g\right)\right\} \leq \alpha_{g} d\left(h, h^{\prime}\right)
\end{aligned}
$$

for all $h, h^{\prime} \in G$. We call a group Lipschitz if there exists a Lipschitz metric on $G$. If $(G, d)$ is a Lipschitz group then we define the distortion of $g$ by

$$
a_{g}:=\inf \left\{\alpha_{g} \geq 1: \alpha_{g} \text { satisfies the condition above for all } h, h^{\prime} \in G\right\} .
$$

We say that a cocycle $f$ has bounded distortion if there exists a constant $c \geq 1$ with $\alpha_{\delta}\left(f\left(T^{n} x\right) \ldots f(x)\right) \leq c$ for $\mu-$ a.e. $x \in X$ and every $n \in \mathbb{Z}$.

In this context we mention the following proposition due to Schmidt [19].

Proposition 4.1. Let $f: X \longmapsto G=G L(n, \mathbb{C})$ be a Borel map such that $\mathbf{f}: \mathbb{Z} \times X \longmapsto$ $G$ has bounded distortion with respect to the natural metric. Then $f$ is cohomologous to a Borel map $g: X \longmapsto U(n)$.

There are many open questions to consider. Is it possible to have a cocycle $g$ which does not satisfy the pinching condition taking values in a semisimple Lie group which satisfies $g(x)=h(T x) h(x)^{-1}$ for a measurable but not continuous cobounding function $h$ ?

One may also ask if Theorem 1.1 holds if the base transformation is an Axiom A diffeomorphism equipped with a Gibbs measure. If Proposition 2.4 can be extended to Axiom A base transformations with Gibbs measures then our main theorem does as well.

Acknowledgements. We would like to thank Sasha Borovik, Dima Dolgopyat, Shahar Mozes, Klaus Schmidt and especially S. G. Dani for interesting and useful discussions. MN would like to thank the Nuffield Foundation for their support.

\section{A. Appendix. Some groups which satisfy Hypothesis 1}

We begin by recalling a result of A. Borel which guarantees the existence of cocompact lattices.

Proposition A.1. ((Borel) [18, Ch. 14]) Let $\widetilde{M}$ be a non-compact symmetric space, then it admits a lattice $\Gamma$ with compact quotient.

S. Mozes and S. G. Dani have suggested the following approach to checking which groups satisfy Hypothesis 1 . Recall that any proper subgroup of $G$ has dimension strictly less than $G$.

Using Borel's theorem with $G=\tilde{M}$, choose any cocompact lattice $\Gamma$. Suppose $\Gamma=$ $\left\{\gamma_{1}, \ldots, \gamma_{n}, \ldots\right\}$ is an enumeration of this lattice. Let $A_{i, j}=\left\{\gamma \in G: \gamma \gamma_{i} \gamma^{-1}=\gamma_{j}\right\}$ and let $C_{G}\left(\gamma_{i}\right)$ denote the centralizer of $\gamma_{i}$ (in the group $G$ ). Note that the dimension of $C_{G}\left(\gamma_{i}\right)$ is strictly less than that of $G$, for each $i$, since $C_{G}\left(\gamma_{i}\right)$ is a proper subgroup of $G$.

We claim that $A_{i, j}=t_{i, j}^{-1} C_{G}\left(\gamma_{i}\right)$ for some $t_{i, j} \in G$. Suppose that $A_{i, j} \neq \varnothing$ and observe that $A_{j, i}=\left\{\gamma^{-1}: \gamma \in A_{i, j}\right\} \neq \emptyset$. Fix an element $t_{i, j} \in A_{j, i}$ and let $\gamma \in A_{i, j}$. 
Note that $\left(t_{i, j} \gamma\right) \gamma_{i}\left(t_{i, j} \gamma\right)^{-1}=t_{i, j} \gamma_{j} t_{i, j}^{-1}=\gamma_{i}$ and hence $t_{i j} \gamma \in C\left(\gamma_{i}\right)$. Hence we see that $\gamma \in t_{i, j}^{-1} C\left(\gamma_{i}\right)$, and our claim follows.

The union $C:=\cup_{1 \leq i, j} A_{i, j}$ consists of all elements in $G$ which conjugate at least one element in $\Gamma$ to another. Since this is a countable union, $C$ again has dimension strictly less than that of $G$.

Fix an element $g \in G-C$, then the subgroup $\Gamma^{\prime}=g^{-1} \Gamma g$ is a cocompact lattice. Furthermore, by construction $\Gamma$ and $\Gamma^{\prime}$ satisfy Hypothesis 1 .

S. G. Dani has made the observation that if the group has a finite centre then the above argument works for lattices containing no non-trivial central elements. Since in a linear semisimple Lie group any lattice contains a subgroup of finite index which is torsion-free (and hence has trivial intersection with the centre), there are always cocompact lattices with trivial intersection with the centre [18, Corollary 6.13, Theorem 6.15 and Remark 6.18]. Thus all linear semisimple Lie groups satisfy Hypothesis 1 .

Unfortunately, this argument fails in the case of the Heisenberg group. Any lattice in a connected linear semisimple Lie group contains a subgroup of finite index in the centre [18, Corollary 5.17]. Hence this will also be true of the intersection of two lattices. Thus if the centre of the group is infinite then the intersection of two lattices cannot be trivial. The Heisenberg group has an infinite centre so the intersection of two cocompact lattices in the Heisenberg group must contain more than the identity element.

\section{REFERENCES}

[1] M. Brin. Ergodic theory of frame flows. Ergodic Theory and Dynamical Systems. Vol. 2. Ed. A. Katok. Birkhaüser, 1982, pp. 163-183.

[2] M. Brin and Y. Pesin. Partially hyperbolic dynamical systems. Math. USSR Izv 8 (1974), 177-218.

[3] Encyclopaedia of Mathematics. Vols 1-6. Ed. M. Hazewinkel. Kluwer Academic Publishers, Dordrecht, 1995.

[4] M. Hirsch, C. Pugh and M. Shub. Invariant manifolds (Springer Lecture Notes in Mathematics, 583). Springer, Berlin, 1977

[5] A. Katok and B. Hasselblatt. An Introduction to the Modern Theory of Dynamical Systems (Encyclopedia of Mathematics and Its Applications, 54). Cambridge University Press, 1997.

[6] A. Katok and A. Kononenko. Cocycle stability for partially hyperbolic systems. Math. Res. Lett. 3 (1996), 191-210.

[7] R. de la Llave. Smooth conjugacy and SRB measures for uniformly and nonuniformly hyperbolic systems. Comm. Math. Phys. 150 (1992), 289-320.

[8] R. de la Llave, J. M. Marco and R. Moriyon. Canonical perturbation theories of Anosov diffeomorphisms and regularity results for the Livšic cohomology equation. Ann. Math. 123 (1987), 537-611.

[9] A. N. Livšic. Cohomology of dynamical systems. Math. USSR Izvestija 6(6) (1972), 1278-1301.

[10] M. Nicol and M. Pollicott. Measurable cocycle rigidity for some noncompact groups. Bull. London Math. Soc. 31 (1999), 592-600.

[11] V. Niţică and A. Török. Regularity results for the solution of the Livšic equation with values in diffeomorphism groups. Ergod. Th. \& Dynam. Sys. 16 (1996), 325-333.

[12] W. Parry. Compact abelian group extensions of discrete dynamical systems. Z. Wahr. Vewr. Geb 13 (1969), 95-113.

[13] W. Parry. Skew products of shifts with a compact Lie group. J. London Math. Soc., 2nd Series 56(2) (1997), 395-404.

[14] W. Parry and M. Pollicott. The Livšic cocycle equation for compact Lie groups of hyperbolic systems. J. London Math. Soc., 2nd series 56 (1997), 405-416. 
[15] M. Pollicott. On the rate of mixing of Axiom A flows. Invent. Math. 81 (1990), 413-426.

[16] C. Pugh and M. Shub. Stably ergodic dynamical systems and partial hyperbolicity. J. Complexity 13(1) (1997), 125-179.

[17] A. Quas. Rigidity of continuous coboundaries. Bull. London Math. Soc. 29(5) (1997), 595-600.

[18] M. Ragunathan. Discrete Subgroups of Lie Groups. Springer, Berlin, 1972.

[19] K. Schmidt. Remarks on Livšics theory for nonabelian cocycles. Ergod. Th. \& Dynam. Sys. 19(3) (1999), 703-721.

[20] C. Walkden. Livšic regularity theorems for twisted cocycle equations over hyperbolic systems. J. London Math. Soc. 2:61(1) (2000), 286-300. 